University of Wollongong

Research Online

Faculty of Engineering and Information

Faculty of Engineering and Information

Sciences - Papers: Part A

Sciences

$1-1-2014$

Defects induced ferromagnetism in plasma-enabled graphene nanopetals

Zengji Yue

University of Wollongong, zy709@uowmail.edu.au

Donghan Seo

The University of Sydney

Kostya Ostrikov

University of Wollongong

Xiaolin Wang

University of Wollongong, xiaolin@uow.edu.au

Follow this and additional works at: https://ro.uow.edu.au/eispapers

Part of the Engineering Commons, and the Science and Technology Studies Commons

Research Online is the open access institutional repository for the University of Wollongong. For further information contact the UOW Library: research-pubs@uow.edu.au 


\title{
Defects induced ferromagnetism in plasma-enabled graphene nanopetals
}

\begin{abstract}
Ferromagnetism in graphene is fascinating, but it is still a big challenge for practical applications due to the weak magnetization. In order to enhance the magnetization, here, we design plasma-enabled graphene nanopetals with ultra-long defective edges of up to $105 \mathrm{~m} / \mathrm{g}$, ultra-dense lattice vacancies, and hydrogen chemisorptions. The designed graphene nanopetals display robust ferromagnetism with large saturation magnetization of up to $2 \mathrm{emu} / \mathrm{g}$ at $5 \mathrm{~K}$ and $1.2 \mathrm{emu} / \mathrm{g}$ at room temperatures. This work identifies the plasma-enabled graphene nanopetals as a promising candidate for graphene-based magnetic devices. 2014 AIP Publishing LLC.
\end{abstract}

\section{Keywords}

defects, graphene, induced, nanopetals, ferromagnetism, plasma, enabled

Disciplines

Engineering | Science and Technology Studies

\section{Publication Details}

YUE, Z., Seo, D., Ostrikov, K. \& Wang, X. (2014). Defects induced ferromagnetism in plasma-enabled graphene nanopetals. Applied Physics Letters, 104 (9), 092417-1-092417-4. 


\section{AIP / Applied Physics \\ Letters}

\section{Defects induced ferromagnetism in plasma-enabled graphene nanopetals}

Z. J. Yue, D. H. Seo, K. Ostrikov, and X. L. Wang

Citation: Applied Physics Letters 104, 092417 (2014); doi: 10.1063/1.4867891

View online: http://dx.doi.org/10.1063/1.4867891

View Table of Contents: http://scitation.aip.org/content/aip/journal/apl/104/9?ver=pdfcov

Published by the AIP Publishing

\section{Articles you may be interested in}

First-principles study of carrier-induced ferromagnetism in bilayer and multilayer zigzag graphene nanoribbons Appl. Phys. Lett. 104, 143111 (2014); 10.1063/1.4870766

Surface defects induced ferromagnetism in mechanically milled nanocrystalline $\mathrm{ZnO}$ J. Appl. Phys. 114, 073516 (2013); 10.1063/1.4818802

Influence of Li-N and Li-F co-doping on defect-induced intrinsic ferromagnetic and photoluminescence properties of arrays of $\mathrm{ZnO}$ nanowires

J. Appl. Phys. 112, 043910 (2012); 10.1063/1.4747929

Defect and adsorbate induced ferromagnetic spin-order in magnesium oxide nanocrystallites

Appl. Phys. Lett. 100, 192404 (2012); 10.1063/1.4712058

Ferromagnetic $\mathrm{ZnO}$ nanocrystals and Al-induced defects

J. Appl. Phys. 110, 114316 (2011); 10.1063/1.3665637

\section{Asylum Research Atomic Force Microscopes}

Unmatched Performance, Versatility and Support

Modulus of Polymers

\& Advanced Materials

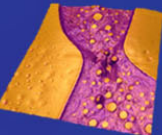

Coating Uniformity \& Roughness

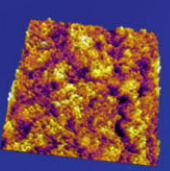

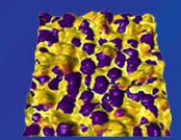

Nanoscale Conductivity \& Permittivity Mapping
Piezoelectrics \& Ferroelectrics

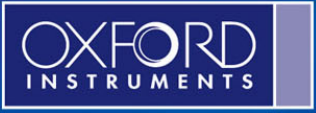

The Business of Science ${ }^{2}$
+1 (805) 696-6466 sales@AsylumResearch.com www.AsylumResearch.com 


\title{
Defects induced ferromagnetism in plasma-enabled graphene nanopetals
}

\author{
Z. J. Yue, ${ }^{1,2}$ D. H. Seo, ${ }^{2,3}$ K. Ostrikov, ${ }^{2,3,1}$ and X. L. Wang ${ }^{1, a)}$ \\ ${ }^{1}$ Institute for Superconducting and Electronic Materials (ISEM), Faculty of Engineering, \\ University of Wollongong, Wollongong, NSW 2522, Australia \\ ${ }^{2}$ Plasma Nanoscience Centre Australia (PNCA), CSIRO Materials Science and Engineering, P. O. Box 218, \\ Lindfield, NSW 2070, Australia \\ ${ }^{3}$ Plasma Nanoscience, School of Physics, The University of Sydney, Sydney, NSW 2006, Australia
}

(Received 19 December 2013; accepted 7 February 2014; published online 6 March 2014)

\begin{abstract}
Ferromagnetism in graphene is fascinating, but it is still a big challenge for practical applications due to the weak magnetization. In order to enhance the magnetization, here, we design plasma-enabled graphene nanopetals with ultra-long defective edges of up to $10^{5} \mathrm{~m} / \mathrm{g}$, ultra-dense lattice vacancies, and hydrogen chemisorptions. The designed graphene nanopetals display robust ferromagnetism with large saturation magnetization of up to $2 \mathrm{emu} / \mathrm{g}$ at $5 \mathrm{~K}$ and $1.2 \mathrm{emu} / \mathrm{g}$ at room temperatures. This work identifies the plasma-enabled graphene nanopetals as a promising candidate for graphene-based magnetic devices. (C) 2014 AIP Publishing LLC. [http://dx.doi.org/10.1063/1.4867891]
\end{abstract}

Magnetic ordering in carbon-based materials has attracted widespread attention as variable light-element alternative to conventional transition metals like $\mathrm{Fe}, \mathrm{Co}, \mathrm{Ni}$, etc. ${ }^{1-4}$ Carbon-based magnetic materials possess a lot of attractive properties, such as low density, biocompatibility, and plasticity, which are practical significance for next generation magnetic devices., ${ }^{5,6}$ Graphene, a two dimensional single sheet of carbon, shows extraordinarily high electron mobility, thermal conductivity, and mechanical strength, and is promising for many applications such as nanoelectronics, spintronics, and optoelectronics. ${ }^{7,8}$ Magnetic ordering in graphenes may be used in the design of future magnetic nanoelectronic and spintronic devices. Ferromagnetism has been predicted and observed in graphite and graphene and is attributed to localized unpaired spins induced by defects. ${ }^{9-13}$ The induced magnetic moments interact ferromagnetically if defects are present at different hexagonal sublattices of graphene. $^{14}$

Many approaches have been used to produce ferromagnetism in graphene, including introduction of hydrogen $(\mathrm{H})$ chemisorptions defects, vacancy defects, and edge defects as well as proximity effects. ${ }^{13,15-18}$ To date, however, the observed maximum magnetization in either polycrystalline or single-crystal graphene synthesized by Chemical Vapor Deposition (CVD) or mechanical cleavage is weak. ${ }^{5,11}$ This is because only a tiny fraction of the carbon atoms in graphene contribute to the magnetism. ${ }^{3}$ The lack of large magnetization hinders the applications of graphene in practical magnetic devices. In order to achieve large magnetization and realize practical applications, it is essential to employ new techniques to enhance intrinsic magnetization of graphenes.

Here, to achieve robust ferromagnetism and large saturation magnetization, we design plasma-enabled graphene nanopetals (PEGN) with ultra-long defective edges, ultra-dense vacancies, and $\mathrm{H}$ chemisorptions on edges and

\footnotetext{
${ }^{\text {a) }}$ Author to whom correspondence should be addressed. Electronic mail: xiaolin@uow.edu.au
}

vacancies. Indeed, the PEGNs display robust ferromagnetism with enhanced saturation magnetization of up to $2 \mathrm{emu} / \mathrm{g}$ at low temperatures and $1.2 \mathrm{emu} / \mathrm{g}$ at room temperatures. The observed ferromagnetism is an intrinsic magnetic behavior of nanopetals and can be attributed to the ultra-long defective edges, plasma-induced vacancy defects, and $\mathrm{H}$ adatoms during growth process. Our results indicate that the plasma aided synthesis is a new way to enhance the ferromagnetism in graphenes for practical applications.

Plasma aided growth has been found widely used for the controlled synthesis of nanostructures. ${ }^{19,20}$ For example, vertical graphene networks can be grown directly with selforganized patterns by the plasma-enhanced CVD (PECVD) without any catalyst and substrate heating. ${ }^{21,22}$ The growth of PEGNs was carried out in an inductively coupled plasma (ICP-CVD, $13.56 \mathrm{MHz}, 1.0 \mathrm{~kW}$ ) reactor. First, Ar gas was fed into the chamber where the plasma was generated at 3.0 Pa with RF power $800 \mathrm{~W}$. Likewise $-50 \mathrm{~V}$ of substrate bias was used to enhance the plasma interaction with Si substrate. After $3 \mathrm{~min}$ of the plasma treatment, a gas mixture of $30 \% \mathrm{CH}_{4}, 20 \% \mathrm{H}_{2}$, and $50 \% \mathrm{Ar}$ was fed into the chamber and the rf power was increased up to $800 \mathrm{~W}$ for the deposition. Here, $\mathrm{H}$ atoms can be adsorbed on graphene surface when $\mathrm{H}$ plasma is exposed to the sample at high temperatures. Hence, the plasma serves as a main $\mathrm{H}$ source and produces hydrogen absorption defects in graphene. ${ }^{23}$ The deposition time was kept for $8 \mathrm{~min}$.

Figure 1(a) shows the scanning electron microscope (SEM) images of PEGNs on $\mathrm{Si}$, in which the graphene nanosheets are all nearly perpendicular to their substrates, with the height ranging from 300 to $500 \mathrm{~nm}$. Based on the scale of SEM images, the estimated edge length is up to $10^{5} \mathrm{~m} / \mathrm{g}$. To ensure the magnetic signal originating from the graphene nanosheets, we have analyzed the graphene nanopetals with X-ray Photoelectron Spectroscopy (XPS) and Energydispersive X-ray Spectroscopy (EDX). As shown in Figs. 1(c) and $1(\mathrm{~d})$, only carbon elements have been detected, which proves that our PEGNs have high purity. 

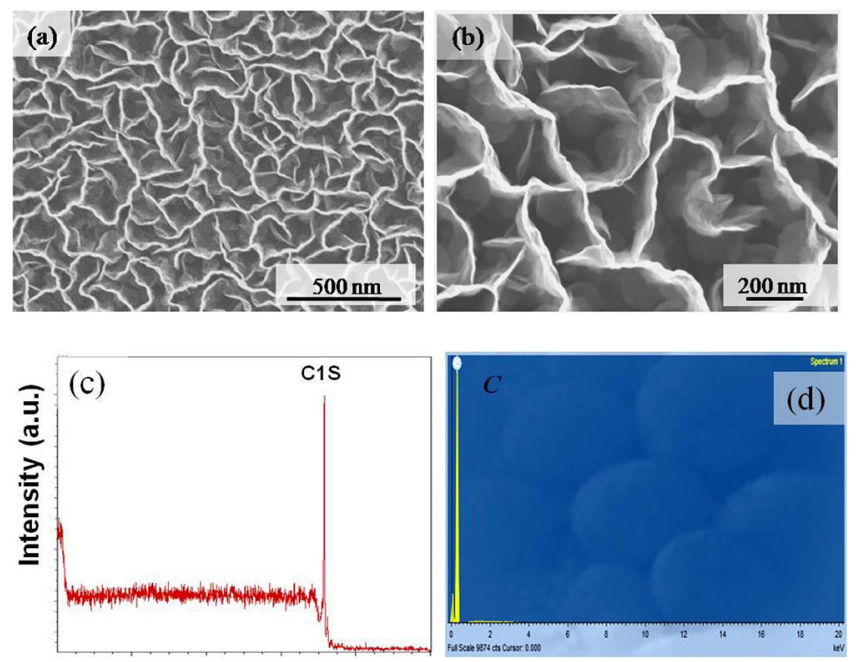

FIG. 1. (a) and (b) SEM images, (c) XPS spectra, and (d) EDX spectra for the PEGNs.

The magnetization analysis of the graphene nanopetal films has been performed with vibrating sample magnetometry (VSM) in Physical Property Measurement System (PPMS). To exclude the influences of $\mathrm{SO}_{2} / \mathrm{Si}$, the PEGNs were cleaved from the substrate before the VSM measurements. First, the sample holder and plastic tapes which were used to carry samples were measured and displayed absolute diamagnetism, as shown in Fig. 2(a). Fig. 2(b) displays the magnetic hysteresis loops of the PEGN powders (milled from PEGNs) measured at temperatures 5, 50, and $200 \mathrm{~K}$, which show weak ferromagnetism at $5 \mathrm{~K}$ and paramagnetism at higher temperatures. The saturation magnetization $M_{s}$ is about $0.6 \mathrm{emu} / \mathrm{g}$ at $200 \mathrm{~K}$. Figures 2(c) and 2(d) exhibit the magnetization hysteresis loops for the PEGNs at 5, 10, 50, 100,200 , and $300 \mathrm{~K}$. The saturation magnetic moment reaches up to $2 \mathrm{emu} / \mathrm{g}$ at $5 \mathrm{~K}$ and $1.2 \mathrm{emu} / \mathrm{g}$ at $300 \mathrm{~K}$. The ferromagnetism can be seen clearly in the enlarged hysteresis loops near zero field in Fig. 2(d).

Figure 3(a) shows the magnetic behavior of PEGNs as a function of temperatures [zero field cooled (ZFC) and field cooled (FC) measurements for $\mathrm{H}=1 \mathrm{kOe}$. A clear magnetic transition was observed at $T_{c}=365 \mathrm{~K}$. The extracted temperature dependence of the saturation magnetization and coercivity are plotted in Figs. 3(b) and 3(c). Compared with the magnetization in PEGN powder, the saturation magnetization is significantly enhanced. This shows that the morphology and structure of PEGNs play a significant role in the generation of robust ferromagnetism and giant saturation magnetization.

Raman spectrum of PEGNs is shown in Fig. 4(a). D peak is located at $1350 \mathrm{~cm}^{-1}, \mathrm{G}$ peak is located at $1580 \mathrm{~cm}^{-1}, \mathrm{D}^{\prime}$ peak is located at $1620 \mathrm{~cm}^{-1}$, and $2 \mathrm{D}$ peak is located at $2700 \mathrm{~cm}^{-1}$. Raman spectrum contains important information about the graphitic structure of our PEGNs. First, high $\mathrm{I}_{\mathrm{D}} / \mathrm{I}_{\mathrm{G}}$ ratio of 2.0 reveals that our PEGNs contain a certain degree of defects in the structure. The presence of $\mathrm{D}^{\prime}$ peak suggests the high possibility of our PEGN is hydrogenated. ${ }^{23}$ The $\mathrm{I}_{\mathrm{D}} / \mathrm{I}_{\mathrm{D}^{\prime}}$ peak ratio of 4.0 suggests that our graphene nanopetals also contain edge defects. ${ }^{24}$

As shown in Fig. 4(b), in our PEGNs, the $\mathrm{H}$ adatoms are not only adsorbed on the graphene surfaces but also at interlayers during the plasma-growth process. ${ }^{25} \mathrm{H}$ adatoms in graphene nanopetals can generate spontaneous magnetism in graphene through forming $\mathrm{C}-\mathrm{H}$ bonds and unpaired electrons. $^{26-29}$ The $\mathrm{H}$ chemisorption defects give rise to strong Stoner ferromagnetism with a magnetic moment of $1 \mu_{B}$ per defect when the defects are located at the same sublattice. $^{30}$ Hydrogen adatoms induce the formation of unpaired electrons which lead to the observed magnetization
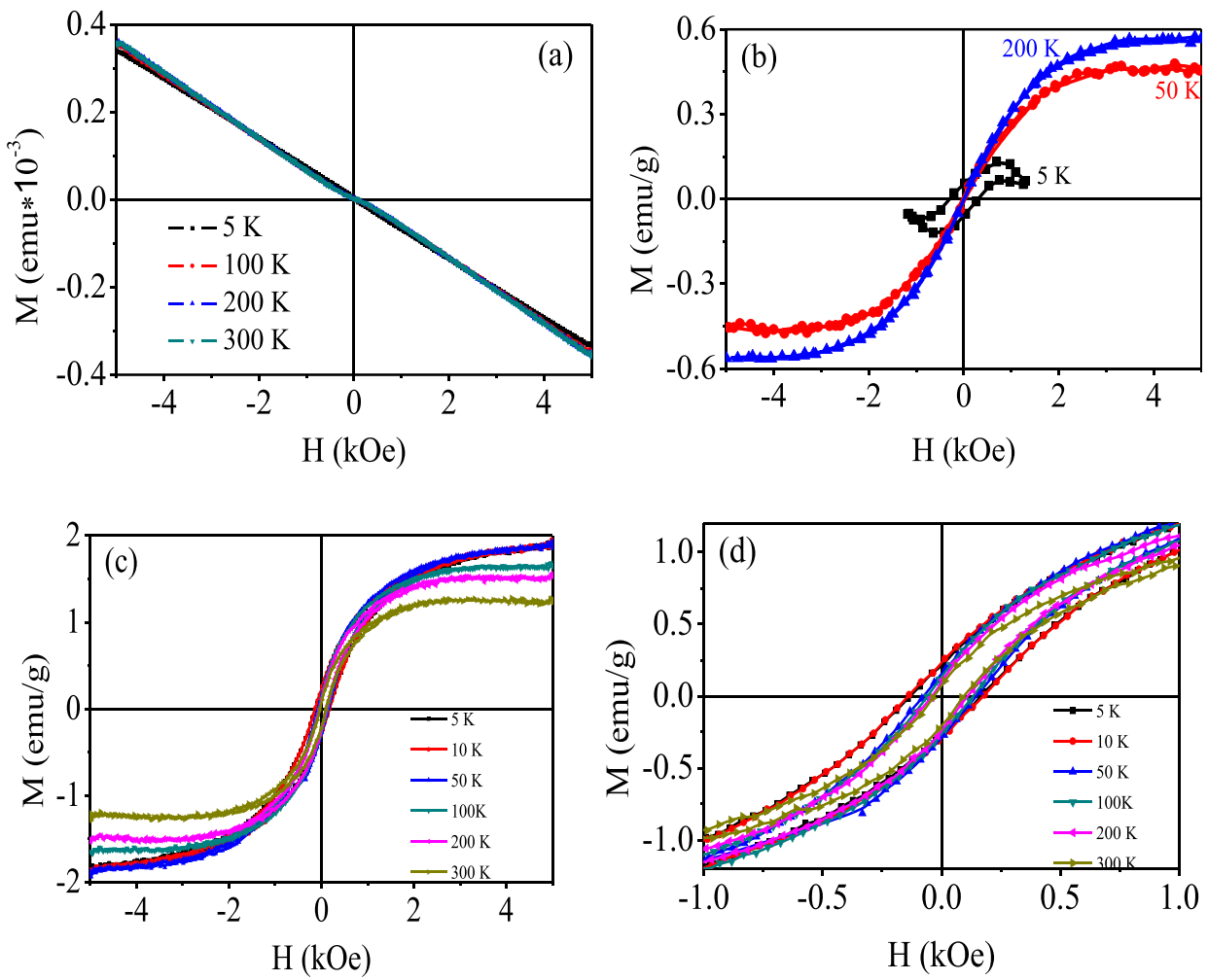

FIG. 2. Magnetization hysteresis loops for (a) sample holder (plastic tube), (b) PEGN powders, (c) and (d) PEGNs at different temperatures. 

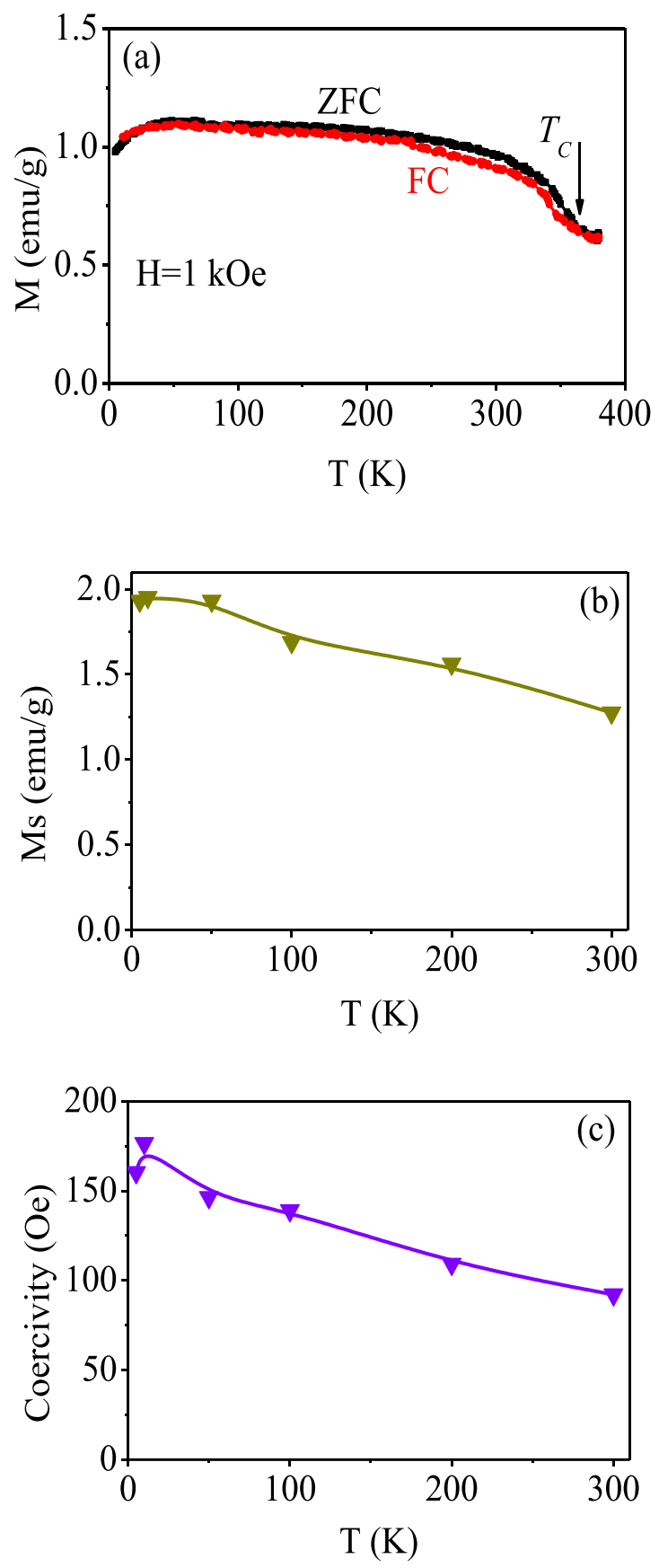

FIG. 3. (a) ZFC and FC magnetization as a function of temperature, ranging from $5 \mathrm{~K}$ to $380 \mathrm{~K}$. The arrow shows the magnetic transition temperatures, $T c=365 \mathrm{~K}$. (b) Saturation magnetization and (c) coercivity as a function of temperatures. in graphene nanopetals. ${ }^{31}$ The stability of the magnetic configurations depends on the distance between $\mathrm{H}$ adatoms and the strength of exchange couplings between the defectinduced magnetic moments. ${ }^{30}$ Additionally, strong longrange coupling between local magnetic moments at the same sublattice of PEGNs can maintain room-temperature ferromagnetic ordering against thermal fluctuations.

The PEGNs have one side with open defective edges and another side attached to the substrate. The zigzag and armchair edges coexist in PEGNs. The zigzag edges can be magnetic due to spin-polarized edge states. ${ }^{28}$ The net spin moment in zigzag-edged nanosheets results from topological frustration of the $\pi$-bonds. ${ }^{32}$ Moreover, the absorption of $\mathrm{H}$ at the edges of PEGNs leads to the formation of a spinpolarized band at the Fermi level. The $\pi$ electrons on hydrogenated zigzag edges of PEGNs may create a ferromagnetic spin structure on the edge due to edge localized states. In addition, $\mathrm{H}$ adatoms at zigzag edges of PEGNs can passivate the $\sigma$ dangling bonds and leave all the $\pi$ orbitals unsaturated and carrying the magnetic moments. The magnitude of the ferromagnetism strongly depends on the position of the $\mathrm{H}$ atoms relative to the edges. ${ }^{33}$ As mentioned above, in the PEGNs, there are ultra-long edges of up to $10^{5} \mathrm{~m} / \mathrm{g}$, which might contribute to the giant saturation magnetization.

Plasma-aided growth of the PEGNs leads to the formation of large amount of atomic vacancies in the graphene nanosheets (Figure 4(b)). These lattice vacancies in PEGNs can generate localized electronic states and magnetic moments due to the hybridization of $p_{z}$ orbitals in the $\pi$-band. Moreover, vacancy defects in the same sublattice of PEGNs can induce ferromagnetic ordering and states in situations of sublattice symmetry breaking. ${ }^{28}$ On the other hand, $\mathrm{H}$ adatoms can easily adsorb on vacancy dangling bonds and form vacancy-H complexes in PEGNs, which might provide larger magnetic moments and the observable macroscopic magnetic signals. ${ }^{34}$

In conclusion, the plasma produced PEGNs exhibit robust ferromagnetic ordering with giant enhanced saturation magnetization. The observed ferromagnetism is much higher than the obtained magnetization in the case of flat few-layer graphene. The PEGNs can be particularly useful to explore magnetic ordering in graphene through introducing controllable edge, lattice vacancy, and hydrogen related defects. By manipulating the plasma conditions, it may be possible to tune the magnetism of graphene. Finally, the PEGNs are very promising candidates for the graphene based magnetic nanodevices.
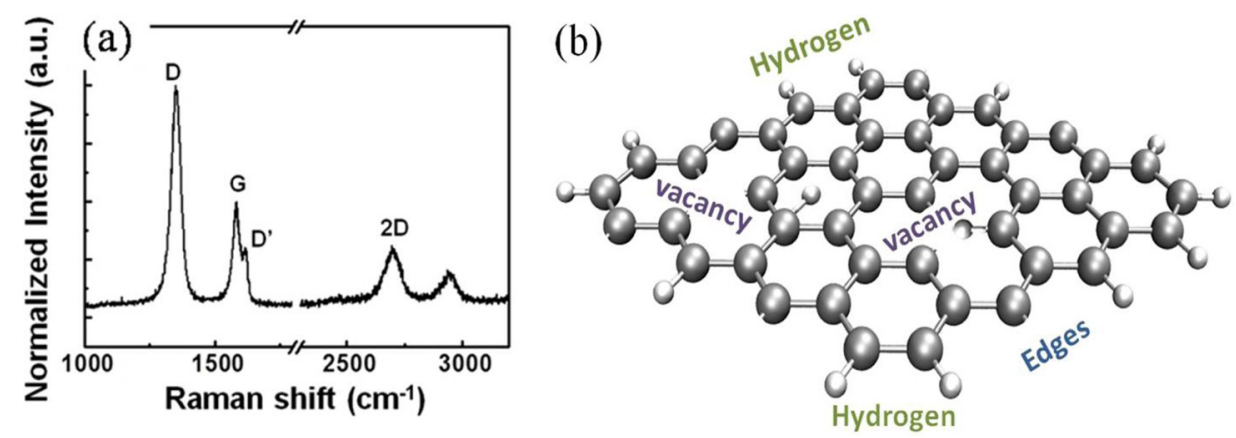

FIG. 4. (a) The Raman spectra of PEGNs that display a relatively high density of defects. (b) Atomic structures of the PEGNs with hydrogen chemisorption defects, edge defects, and lattice vacancy defects. 
This work was partially supported by the Australian Research Council under Discovery Project No. DP1094073 and Future Fellowship Project No. FT100100303. Fruitful discussions with Dr. S. Kumar, Dr. Z. J. Han, Dr. I. Levchenko, and Dr. F. Hong are gratefully acknowledged.

${ }^{1}$ R. C. Haddon, Nature 378, 249 (1995).

${ }^{2}$ T. L. Makarova, B. Sundqvist, R. Hohne, P. Esquinazi, Y. Kopelevich, P. Scharff, V. A. Davydov, L. S. Kashevarova, and A. V. Rakhmanina, Nature 413, 716 (2001)

${ }^{3}$ J. M. D. Coey, M. Venkatesan, C. B. Fitzgerald, A. P. Douvalis, and I. S. Sanders, Nature 420, 156 (2002).

${ }^{4}$ L. Krusin-Elbaum, D. M. Newns, H. Zeng, V. Derycke, J. Z. Sun, and R. Sandstrom, Nature 431, 672 (2004).

${ }^{5}$ O. V. Yazyev, Rep. Prog. Phys. 73, 056501 (2010).

${ }^{6}$ D. Seo, Z. Yue, X. Wang, I. Levchenko, S. Kumar, S. Dou, and K. Ostrikov, Chem. Commun. 49, 11635 (2013).

${ }^{7}$ A. H. Castro Neto, F. Guinea, N. M. R. Peres, K. S. Novoselov, and A. K. Geim, Rev. Mod. Phys. 81, 109 (2009).

${ }^{8}$ M. O. Goerbig, Rev. Mod. Phys. 83, 1193 (2011).

${ }^{9}$ J. Cervenka, M. I. Katsnelson, and C. F. J. Flipse, Nat. Phys. 5, 840 (2009).

${ }^{10}$ C. S. Rout, A. Kumar, N. Kumar, A. Sundaresan, and T. S. Fisher, Nanoscale 3, 900 (2011).

${ }^{11}$ Y. Wang, Y. Huang, Y. Song, X. Zhang, Y. Ma, J. Liang, and Y. Chen, Nano Lett. 9, 220 (2009).

${ }^{12}$ P. Esquinazi, D. Spemann, R. Höhne, A. Setzer, K. H. Han, and T. Butz, Phys. Rev. Lett. 91, 227201 (2003).

${ }^{13}$ H. Xia, W. Li, Y. Song, X. Yang, X. Liu, M. Zhao, Y. Xia, C. Song, T.-W. Wang, D. Zhu, J. Gong, and Z. Zhu, Adv. Mater. 20, 4679 (2008).

${ }^{14}$ O. V. Yazyev and L. Helm, Phys. Rev. B 75, 125408 (2007).

${ }^{15}$ A. V. Krasheninnikov and F. Banhart, Nature Mater. 6, 723 (2007).
${ }^{16}$ A. Ney, P. Papakonstantinou, A. Kumar, N.-G. Shang, and N. Peng, Appl. Phys. Lett. 99, 102504 (2011).

${ }^{17}$ D. Soriano, N. Leconte, P. Ordejón, J.-C. Charlier, J.-J. Palacios, and S. Roche, Phys. Rev. Lett. 107, 016602 (2011).

${ }^{18}$ H. X. Yang, A. Hallal, D. Terrade, X. Waintal, S. Roche, and M. Chshiev, Phys. Rev. Lett. 110, 046603 (2013).

${ }^{19}$ E. Tam, I. Levchenko, and K. Ostrikov, J. Appl. Phys. 100, 036104 (2006).

${ }^{20}$ K. Ostrikov, E. C. Neyts, and M. Meyyappan, Adv. Phys. 62, 113 (2013).

${ }^{21}$ D. H. Seo, S. Kumar, and K. Ostrikov, Carbon 49, 4331 (2011).

${ }^{22}$ Z. Yue, I. Levchenko, S. Kumar, D. Seo, X. Wang, S. Dou, and K. Ostrikov, Nanoscale 5, 9283 (2013).

${ }^{23}$ D. C. Elias, R. R. Nair, T. M. G. Mohiuddin, S. V. Morozov, P. Blake, M. P. Halsall, A. C. Ferrari, D. W. Boukhvalov, M. I. Katsnelson, A. K. Geim, and K. S. Novoselov, Science 323, 610 (2009).

${ }^{24}$ A. Eckmann, A. Felten, A. Mishchenko, L. Britnell, R. Krupke, K. S. Novoselov, and C. Casiraghi, Nano Lett. 12, 3925 (2012).

${ }^{25}$ Y.-W. Son, M. L. Cohen, and S. G. Louie, Nature 444, 347 (2006).

${ }^{26}$ K. M. McCreary, A. G. Swartz, W. Han, J. Fabian, and R. K. Kawakami, Phys. Rev. Lett. 109, 186604 (2012).

${ }^{27}$ P. Ruffieux, O. Gröning, M. Bielmann, P. Mauron, L. Schlapbach, and P. Gröning, Phys. Rev. B 66, 245416 (2002).

${ }^{28}$ H.-X. Yang, M. Chshiev, D. W. Boukhvalov, X. Waintal, and S. Roche, Phys. Rev. B 84, 214404 (2011).

${ }^{29}$ J. Zhou, Q. Wang, Q. Sun, X. S. Chen, Y. Kawazoe and P. Jena, Nano Lett. 9, 3867 (2009).

${ }^{30}$ A. Ranjbar, M. S. Bahramy, M. Khazaei, H. Mizuseki, and Y. Kawazoe, Phys. Rev. B 82, 165446 (2010).

${ }^{31}$ L. Xie, X. Wang, J. Lu, Z. Ni, Z. Luo, H. Mao, R. Wang, Y. Wang, H. Huang, D. Qi et al., Appl. Phys. Lett. 98, 193113 (2011).

${ }^{32}$ W. L. Wang, S. Meng, and E. Kaxiras, Nano Lett. 8, 241 (2008).

${ }^{33}$ D. Soriano, F. Muñoz-Rojas, J. Fernández-Rossier, and J. J. Palacios, Phys. Rev. B 81, 165409 (2010)

${ }^{34}$ P. O. Lehtinen, A. S. Foster, Y. Ma, A. V. Krasheninnikov, and R. M. Nieminen, Phys. Rev. Lett. 93, 187202 (2004). 